\title{
Measurement of blood pressure in the leg-a statement on behalf of the British and Irish Hypertension Society
}

\author{
James P. Sheppard ${ }^{1}$. Peter Lacy ${ }^{2}$ Philip S. Lewis ${ }^{3}$ Una Martin ${ }^{4}$ on behalf of the Blood Pressure Measurement \\ Working Party of the British and Irish Hypertension Society
}

Received: 17 April 2019 / Revised: 5 February 2020 / Accepted: 28 February 2020 / Published online: 22 April 2020

(c) The Author(s) 2020. This article is published with open access

\section{Executive summary}

Ankle blood pressure (BP) measurement is necessary for the diagnosis of hypertension where measurements are not possible due to medical conditions or limb deformities. Based on a recent review of the evidence, we recommend an ankle BP threshold of $\geq 155 / 90 \mathrm{mmHg}$ to define high blood pressure in patients who do not have vascular disease. We recommend that ankle BP readings are taken with the subject lying down, using a validated automated device with the cuff placed around the ankle/lower calf.

\section{Full statement}

Blood pressure (BP) is normally measured on the upper arm, but occasionally this is not possible. The presence of fractures, wounds, vascular access devices and shunts, morbid obesity, surgical procedures, lymphoedema, limb deformities (phocomelia) and amputations may prevent the satisfactory cuff placement around the upper arm. In addition, BP measurement may be inaccurate in the presence of

Members of the Blood Pressure Measurement Working Party of the British and Irish Hypertension Society are listed below

Acknowledgements.

British and Irish Hypertension Society

bihs@in-conference.org.uk

1 Nuffield Department of Primary Care Health Sciences, University of Oxford, Oxford, UK

2 Population Science \& Experimental Medicine, Institute of Cardiovascular Science, University College London, London, UK

3 Stockport NHS Foundation Trust, Stockport and University of Manchester, Manchester, UK

4 School of Pharmacy, University of Birmingham, Birmingham, UK bilateral subclavian artery stenoses such as can occur with Takayasu's arteritis [1] or atherosclerosis [2]. In these circumstances, measurement of $\mathrm{BP}$ in the leg may be necessary. It is important to recognise, however, that BP measurements in the arm may differ from those in the legs.

A recent systematic review examined the relationship between supine BP measurements in the arm and leg [3]. A review of 44 studies involving 9771 patients concluded that ankle systolic BP was on average $17.0 \mathrm{mmHg}(95 \% \mathrm{CI}$ 15.4-21.3 mmHg) higher than arm systolic BP, whilst there was no difference in diastolic BP in the general population [3]. These findings suggest that a threshold of $\geq 155 / 90$ $\mathrm{mmHg}$ could be used for diagnosing hypertension in routine practice when only ankle measurements are available. This threshold is conservative and would ensure maximum sensitivity to detect hypertension at the expense of some specificity.

It should be noted that the review found much lower leg pressures in the presence of peripheral vascular disease (PVD). The proposed threshold should therefore be used with caution, and patients with low ankle BPs in the presence of cardiovascular risk factors (e.g. diabetes, renal disease and existing cardiovascular disease) should be considered for further investigation, especially if there is a history of intermittent claudication or clinical evidence of PVD (e.g. femoral arterial bruits, poor or absent foot pulses, poor distal skin perfusion, cold peripheries or arterial ulceration). In such cases, arterial Doppler ultrasonography, CT or MR angiography can be used to confirm significant PVD which may invalidate the use of ankle BP as a surrogate for arm BP. PVD may be worse in one leg compared with the other, so ankle BPs should be taken in both legs where this is suspected.

The review found no consistent or accepted method for measuring BP in the leg. We therefore propose that ankle $\mathrm{BP}$ is measured in a supine position, using a cuff placed around the ankle/lower calf (Fig. 1), ensuring the bladder encircles $\geq 80 \%$ of the ankle circumference. Readings 


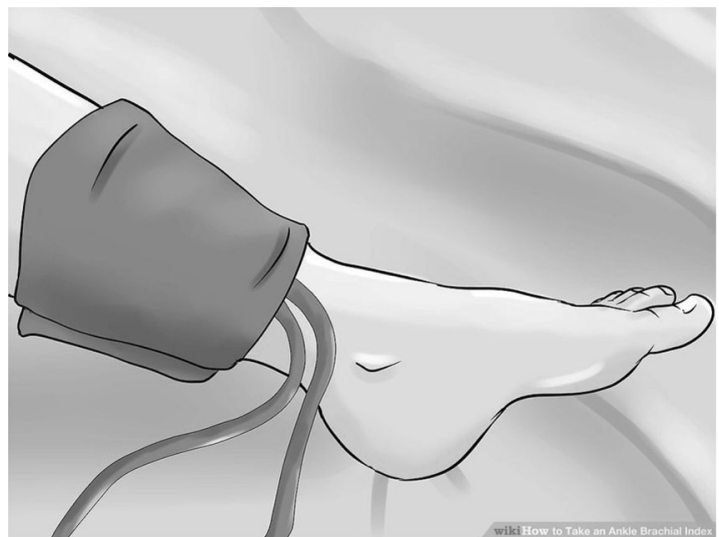

Fig. 1 Positioning of the blood pressure monitor cuff on the ankle/ lower calf. Image taken from Wiki How to take an Ankle Brachial Index (https://www.wikihow.com/Take-an-Ankle-Brachial-Index).

should be taken either by oscillometry or Doppler readings of return to flow at the dorsalis pedis or posterior tibial arteries (systolic readings only). Auscultation is not feasible in most subjects and is not therefore recommended. Ankle BPs are recommended rather than calf or thigh measurements because they generally cause less discomfort and the cuff is easier to fit, particularly in obese patients. As with standard clinic BP measurement, readings should be taken after a 5-min rest period [4]. In terms of oscillometric BP monitors, these have not been specifically validated for leg measurements but are widely used in clinical practice and are a reasonable choice. It is important to note that the use of ambulatory readings for diagnosis will not be possible in patients requiring leg BP measurements. However, where out-of-office measurements are required, home ankle BP monitoring could be considered after appropriate training.

Ankle BP measurement represents a viable alternative to arm measurement for the diagnosis of hypertension, where placement of a cuff on the upper arm is not possible. A threshold of $\geq 155 / 90 \mathrm{mmHg}$ can be recommended, but physicians should use it with caution, recognising that ankle BP measurements may differ significantly in patients with PVD. Given the impracticalities of taking ambulatory measurements in the ankle, we recommend that diagnosis is confirmed and treatment initiated only following consistently high ankle BP readings from repeated clinic visits.

Funding JS receives funding from the Wellcome Trust/Royal Society via a Sir Henry Dale Fellowship (ref: 211182/Z/18/Z). He also receives funding from the NIHR School for Primary Care Research and the NIHR Collaboration for Leadership in Applied Health
Research and Care Oxford at Oxford Health NHS Foundation Trust. PSL receives funding from the NIHR Greater Manchester CRN.

Blood Pressure Measurement Working Party of the British and Irish Hypertension Society N. Chapman ${ }^{5}$, P. Chowienczyk ${ }^{6}$, C. Clark ${ }^{7}$, S. McDonagh ${ }^{7}$, E. Denver ${ }^{8}$, R. McManus ${ }^{9}$, A. Neary ${ }^{10}$

${ }^{5}$ Imperial College London, London, UK; ${ }^{6}$ King's College London, London, UK; ${ }^{7}$ University of Exeter, Exeter, UK; ${ }^{8}$ Whittington Health NHS Trust, London, UK; ${ }^{9}$ University of Oxford, Oxford, UK;

${ }^{10}$ Galway Clinic, Galway, Ireland

\section{Compliance with ethical standards}

Conflict of interest The authors declare that they have no conflict of interest.

Publisher's note Springer Nature remains neutral with regard to jurisdictional claims in published maps and institutional affiliations.

Open Access This article is licensed under a Creative Commons Attribution 4.0 International License, which permits use, sharing, adaptation, distribution and reproduction in any medium or format, as long as you give appropriate credit to the original author(s) and the source, provide a link to the Creative Commons license, and indicate if changes were made. The images or other third party material in this article are included in the article's Creative Commons license, unless indicated otherwise in a credit line to the material. If material is not included in the article's Creative Commons license and your intended use is not permitted by statutory regulation or exceeds the permitted use, you will need to obtain permission directly from the copyright holder. To view a copy of this license, visit http://creativecommons. org/licenses/by/4.0/.

\section{References}

1. Hafner F, Froehlich H, Gary T, Tiesenhausen K, Scarpatetti M, Brodmann M. Blood pressure measurements in patients with Takayasu arteritis: a work of caution. Ann Thorac Surg. 2012;93:1299-301.

2. Aboyans V, Kamineni A, Allison MA, McDermott MM, Crouse $\mathrm{JR}$, Ni H, et al. The epidemiology of subclavian stenosis and its association with markers of subclinical atherosclerosis: the MultiEthnic Study of Atherosclerosis (MESA). Atherosclerosis. 2010;211:266-70.

3. Sheppard JP, Albasri A, Franssen M, Fletcher B, Pealing L, Roberts $\mathrm{N}$, et al. Defining the relationship between arm and leg blood pressure readings: a systematic review and meta-analysis. J Hypertens. 2019;37:660-70.

4. Whelton PK, Carey RM, Aronow WS, Casey DE Jr, Collins KJ, Dennison Himmelfarb C, et al. 2017 ACC/AHA/AAPA/ABC/ ACPM/AGS/APhA/ASH/ASPC/NMA/PCNA guideline for the prevention, detection, evaluation, and management of high blood pressure in adults: executive summary: a report of the American College of Cardiology/American Heart Association Task Force on clinical practice guidelines. Hypertension. 2018;71:1269-324. 